\title{
Mnemonic Discrimination and Social Anxiety: The Role of State Anxiety
}

\author{
Gabriella T. Ponzini \\ West Virginia University, gp0021@mix.wvu.edu
}

Follow this and additional works at: https://researchrepository.wvu.edu/etd

Part of the Clinical Psychology Commons

\section{Recommended Citation}

Ponzini, Gabriella T., "Mnemonic Discrimination and Social Anxiety: The Role of State Anxiety" (2020). Graduate Theses, Dissertations, and Problem Reports. 7659.

https://researchrepository.wvu.edu/etd/7659

This Thesis is protected by copyright and/or related rights. It has been brought to you by the The Research Repository @ WVU with permission from the rights-holder(s). You are free to use this Thesis in any way that is permitted by the copyright and related rights legislation that applies to your use. For other uses you must obtain permission from the rights-holder(s) directly, unless additional rights are indicated by a Creative Commons license in the record and/ or on the work itself. This Thesis has been accepted for inclusion in WVU Graduate Theses, Dissertations, and Problem Reports collection by an authorized administrator of The Research Repository @ WVU. For more information, please contact researchrepository@mail.wvu.edu. 
Mnemonic Discrimination and Social Anxiety: The Role of State Anxiety

Gabriella T. Ponzini, B.A.

Thesis submitted to Eberly College of Arts and Sciences at West Virginia University in partial fulfillment of the requirements for the degree of Master of Science in Psychology

\author{
Shari Steinman, Ph.D. (Chair) \\ Natalie Shook, Ph.D. \\ Claire St. Peter, Ph.D. \\ Department of Psychology
}

Morgantown, West Virginia

2019

Keywords: social anxiety, mnemonic discrimination, state anxiety, episodic memory

Copyright 2019 Gabriella Ponzini 


\section{Abstract \\ Mnemonic Discrimination and Social Anxiety: The Role of State Anxiety}

\section{Gabriella T. Ponzini}

The Mnemonic Similarity Task (MST) measures mnemonic discrimination, or the ability to correctly identify new stimuli from highly similar, old stimuli. Neuroscientific and theoretical suppositions suggest that poor mnemonic discrimination may represent a potential risk or maintenance factor for anxious individuals. However, state affect appears to moderate the relation between mnemonic discrimination abilities and trait anxiety. The current study aimed to elucidate the nascent research on mnemonic discrimination and anxiety by evaluating the MST in a specific subtype of anxiety (i.e., social anxiety) and utilizing a clinically relevant stressor task (i.e., knowledge of a future speech). Participants $(N=131)$ were recruited based on their high or low-levels of social anxiety and were randomly assigned to a stressor condition (i.e., learning about the delivery of a future speech) or a control condition prior to the MST. Participants' levels of self-reported state anxiety were measured throughout the study. Results did not indicate any significant effects related to social anxiety group (high vs. low social anxiety) or condition (stressor vs. control) on mnemonic discrimination abilities. However, this may have been due to the instability of the stressor manipulation or generally low levels of state anxiety across timepoints. Implications or results and future directions are discussed. 


\section{Acknowledgments}

Gabriella would like to thank Dr. Shauna Stark for her expertise and willingness to provide resources and guidance for the MST. She would also like to thank her family, friends, and mentor for their continued encouragement and support! 


\section{Table of Contents}

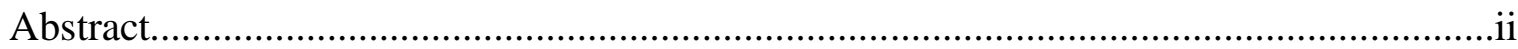

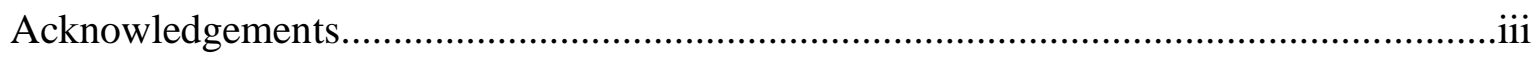

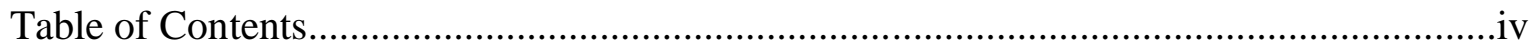

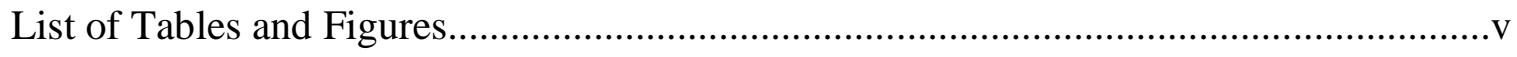

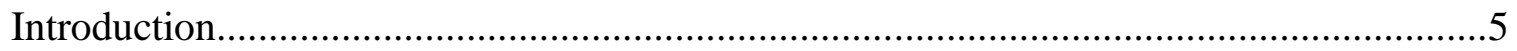

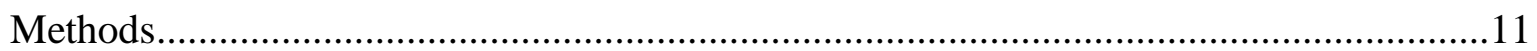

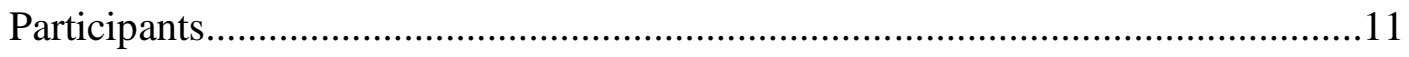

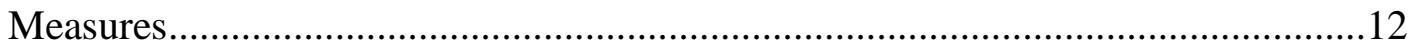

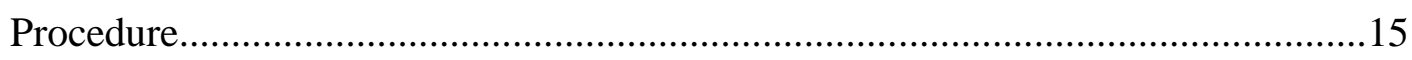

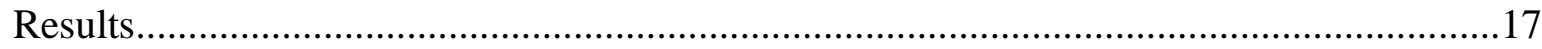

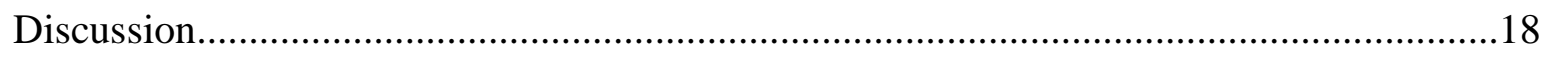

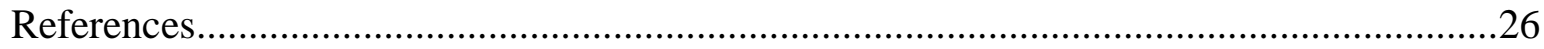

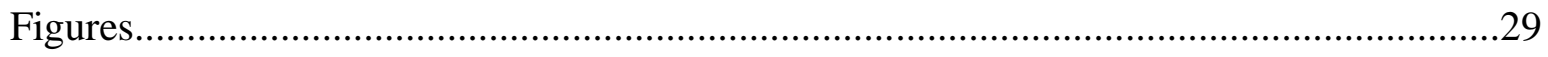

Figure 1: Means and Standard Deviations for LDI Scores Across Study Groups................29

Figure 2: Means and Standard Errors for SUDS Scores by Condition...............................30

Figure 3: Means and Standard Errors for SUDS Scores by Group................................. 31 
Mnemonic Discrimination and Social Anxiety: The Role of State Anxiety

\section{Mnemonic Discrimination and the Mnemonic Similarity Task}

Over the past decade, the mnemonic similarity task (MST) has been used in over 100 publications in populations varying in age (Stark, Stevenson, Wu, Rutledge, \& Stark, 2015; Stark, Yassa, Lacy, \& Stark, 2013; Yassa, Mattfeld, Stark, \& Stark, 2011) and clinical status (Bakker et al., 2012; Kirwan et al., 2012; Yassa et al., 2010) to assess the behavioral impacts associated with hippocampal dysfunction (Kirwan \& Stark, 2007; Stark, Kirwan, \& Stark, 2019). The MST is a modified object recognition task delivered via a computer that consists of two phases: the encoding phase and the retrieval phase. During the encoding phase, participants are shown photographs of indoor and outdoor items and are asked to categorize them accordingly. During the retrieval phase, participants are asked to categorize photographs as new (i.e., "foils"), old (i.e., "targets"), or similar (i.e., "lures"). Notably, the MST differs from traditional recognition tasks (i.e., differentiating "new" from "old” stimuli) by including similar lures. In accordance, researchers who use the MST are particularly interested in the rates for and ways in which participants respond to lure photographs. Mnemonic discrimination abilities are indicated by one's capacity to correctly identify a new entity from one that has been previously seen and is highly similar. On the MST, this involves correctly identifying lures as "similar" while avoiding the tendency to identify them as "old."

MST lure items have been assessed for their degree of similarity via rates of correct versus incorrect identification (i.e., lure items with high mnemonic similarity were photographs more often categorized as "old" than "similar"; Lacy et al., 2011). These data were then used to develop pre-defined stimulus sets for the MST to ensure a balanced number of lure photographs (that vary in their degree of similarity) presented to all participants who take the task. 
"Lure photographs were chosen to be "similar" based on whether they shared the same verbal label (i.e., both apples, bikes, etc.) [as previously seen photographs ("targets")]. We deliberately chose items that varied across multiple dimensions (i.e., size, shape, orientation, etc.). In this way, participants did not learn to only focus on one stimulus feature (e.g., color) to accomplish the task." (S. Stark, personal communication, September 9th, 2019)

In doing so, the MST was designed to place demands on and evaluate behaviors consistent with pattern separation (Kheirbek \& Hen, 2014; Kirwan \& Stark, 2007; Sahay et al., 2011). Pattern separation is a neural computation that occurs in the hippocampus and, when successful, allows for the encoding of detailed episodic memories so that minimal differences register as distinct (Yassa \& Stark, 2011). However, pattern separation processes can fail, and when this occurs, stimuli can overlap in their neural representations (causing memory interference). By introducing similar lure items, researchers can behaviorally assess participants' abilities to preserve unique details about stimuli (which is suggested to rely on pattern separation). The varying levels of similarity of lure stimuli were designed to place increasing demands on pattern separation (Lacy et al., 2011).

The MST has been demonstrated to be a robust, sensitive, and reliable measure of hippocampal functioning in various populations. Functional magnetic resonance imaging (fMRI) studies have shown correlations between poorer mnemonic discrimination abilities and hyperactive blood-oxygen-level-dependent (BOLD) fMRI responses in the dentate gyrus and CA3 subfields in those with hippocampal damage (Kirwan et al., 2012) and cognitive impairment (Yassa et al., 2010; Stark, Yassa, Lacy, \& Stark, 2013). The MST also captures agerelated decline in hippocampal functioning via demonstrations of poorer mnemonic 
discrimination abilities across the aging spectrum (which, traditional recognition tasks are not capable of demonstrating; Stark, Yassa, Lacy, \& Stark, 2013). Importantly, psychological disorders also negatively impact hippocampal structure and functioning (DeCarolis \& Eisch, 2010), and research has demonstrated negative relations between depressive (Shelton \& Kirwan, 2013) and schizophrenic (Das, Ivleva, Wagner, Stark, \& Tamminga, 2014) symptoms and mnemonic discrimination abilities. Corresponding fMRI data suggests that depressive symptoms and episodes of psychosis are associated with impaired activity in the abovementioned hippocampal regions (Fujii et al., 2014; Kraguljac et al., 2018). The MST is also robust against practice effects (Clemenson et al., 2019; Clemenson \& Stark, 2015; Stark et al., 2015), making it an ideal task for assessing change associated with interventions.

\section{Mnemonic Discrimination and Anxiety Symptomatology}

Theoretical and basic science models suggest poor mnemonic discrimination as a potential risk or maintenance factor for anxious individuals (Balderston et al., 2017; Bernstein, Kleinman, \& McNally, 2019; Bernstein \& McNally, 2018; Khierbek et al., 2012; Segal et al., 2012). In particular, fMRI and basic science research indicate that individuals with severe anxiety show structural and functional deficits in the hippocampus (Bannerman, Rawlins, McHugh, Deacon, \& Feldon, 2004; Gray, 1987) that map on to the areas seen in individuals with deficits in pattern separation and poor mnemonic discrimination (DeCarolis \& Eisch, 2010).

Further, a central feature of anxiety disorders is the increasing tendency for individuals to interpret stimuli in their environments and physical experiences as threatening. Etiological and maintenance models of anxiety posit that fear is often generalized from a conditioned stimulus to similar stimuli, events, objects, and situations (Dymond et al., 2015). In other words, fear is experienced when a novel stimulus or sensation evokes a memory of a similar and previously 
learned threatening entity. Although generalization is often seen as adaptive, overgeneralization is thought to be representative of pathological anxiety. Thus, as the generalization of fear broadens, non-threatening stimuli (that grow more dissimilar to the originally learned threat) are interpreted as threatening, eliciting a fear response (American Psychiatric Association, 2013; Craske et al., 2009; Dymond et al., 2015, Dunsmoor \& Paz, 2015; Lissek, 2012). In relation to the MST, healthy participants have a difficult time discriminating only the most similar lure photographs (Lacy et al., 2011); however, based on this theory, anxious individuals should find the discrimination of less similar lure photographs challenging (i.e., indicating poorer mnemonic discrimination abilities).

\section{State Affect and Mnemonic Discrimination}

While research on the relations between anxiety and mnemonic discrimination is in its infancy, to date, studies have not found mnemonic discrimination abilities to predict trait anxiety on their own (Bernstein, Kleinman, \& McNally, 2019; Bernstein \& McNally, 2018). Rather, the individual's affective state seems to moderate the relationship between their symptoms and mnemonic discrimination abilities. In previous studies, affective state has been measured via arousal and valence items from the Self-Assessment Manikin (i.e., visual icons that correlate to participants' current emotional states; Lang, Greenwald, Bradley, \& Hamm, 1993). Specifically, Bernstein and McNally (2018) found that poor performance on the MST predicted anxiety levels (specifically, trait worry) only when participants reported higher levels of state negative affect (i.e., averaged levels of high arousal and negative valence). Similarly, in a subsequent study, authors randomly assigned participants to undergo a modified Trier Social Stress Test (Kirschbaum, Pirke, \& Hellhammer, 1993) where participants prepared and delivered a 5-minute speech (pre-MST encoding) and then completed a 5-minute serial subtraction task (pre-MST 
retrieval). Results suggested that in participants under 40 years old, those who reported higher levels of state negative affect and had higher trait worry (in the stressor condition) had poorer mnemonic discrimination abilities than those in the control condition (Bernstein, Kleinman, \& McNally, 2019).

Mnemonic discrimination is also negatively affected by fear-inducing photographs (Segal et al., 2012) and threats of shock at encoding and retrieval (Balderston et al., 2017). If mnemonic discrimination abilities are negatively affected by affective states, then individuals who frequently experience state anxiety (i.e., situational stress congruent with trait anxiety; Endler \& Kocovski; 2001) may be at a greater risk for mnemonic discrimination impairments (Bernstein, Kleinman, \& McNally, 2019). As such, Bernstein and colleagues (2019) call for research that focuses on specific subtypes of anxiety and emphasizes the emotional context of the anxiety disorder to provide greater evidence for the relations between these constructs.

\section{State Anxiety and Social Anxiety Disorder}

Social anxiety disorder (SAD), or the persistent and debilitating fear of being negatively evaluated in social situations (APA, 2013), represents an appropriate avenue for this research. First, SAD is the most common anxiety disorder (Ruscio et al., 2008), and lifetime prevalence rates are upwards of $12 \%$ in the U.S. population (Stein et al., 2017). Moreover, SAD represents a clinical disorder marked by anticipatory anxiety, or the tendency to experience intense worry about upcoming socially evaluative situations. Given the specific contexts in which these anxiety symptoms occur, manipulating state anxiety via a socially evaluative stressor task is straightforward and common for the social anxiety literature (i.e., primes or behavioral avoidance tasks). The anticipation of public speaking is also the most commonly feared social 
situation by both socially anxious and healthy individuals (thus allowing for a stressor task that negatively affects a wide range of individuals; Mannuzza et al., 1995).

Consistent with theoretical and neuroscientific research associated with mnemonic discrimination, individuals with SAD have been shown to have impairments in their general discrimination abilities for danger and safety cues (Aherns et al., 2016; Ahrens, Mühlberger, Pauli, \& Wieser, 2014; Hermann, Ziegler, Birbaumer, \& Flor, 2002; Sachs, Anderer, Doby, Saletu, \& Dantendorfer, 2003) and some research suggests decreased hippocampal volumes compared to healthy individuals (Irle et al., 2010; Liao et al., 2011; Qiu et al., 2011). Taken together, the theory and research presented herein indicate that assessing the role of mnemonic discrimination in individuals with high levels of social anxiety represents an important next step to help elucidate relations between mnemonic discrimination abilities and state anxiety.

\section{Current Study}

The goal of the present study is to further research on the MST and anxiety by focusing on a specific type of anxiety (i.e., social anxiety) and a clinically relevant stressor task (i.e., learning about the delivery of a future speech; see the review by Wong, 2016 for similar tasks). As such, participants in the present study had high and low levels of social anxiety and were randomized to a stressor condition (i.e., knowledge of a future speech) or control condition.

All hypotheses were preregistered with OSF (https://osf.io/gc2d8). The main hypotheses included: 1) A main effect of stressor condition, such that participants who were randomly assigned to the stressor conditions would perform worse on the MST than the control conditions. This hypothesis is consistent with studies demonstrating the negative impact of stressors on mnemonic discrimination abilities (Balderston et al., 2017; Bernstein, Kleinman, \& McNally, 2019; Segal et al., 2012); 2) An interaction effect, such that for individuals randomly assigned to 
the stressor conditions, those with high levels of social anxiety would perform worse on the MST than those with low levels of social anxiety. This hypothesis is consistent with findings from previous research demonstrating the moderating role of state affect on mnemonic discrimination abilities for individuals with high levels of trait anxiety (Bernstein, Kleinman, \& McNally, 2019; Bernstein \& McNally, 2018). Of note, we did not hypothesize a main effect of social anxiety group due to previous data suggesting that anxiety symptoms alone do not predict MST performance (Bernstein, Kleinman, \& McNally, 2019; Bernstein \& McNally, 2018).

\section{Methods}

\section{Participants}

Participants $(N=154)$ were recruited from West Virginia University between April of 2019 and October of 2019 via SONA (subject pool software for universities), flyers, and email listservs. Eligible participants were between the ages of 18 and 35 and scored either a six or above on the Mini Social Phobia Inventory (Mini-SPIN; indicating clinically elevated levels of social anxiety; Connor et al., 2001) or a three or below on the Mini-SPIN (indicating low levels of social anxiety; Seeley-Wait, Abbott, \& Rapee, 2009). Cut scores were determined by rounding one standard deviation above and below the means of healthy $(M=1.8 ; S D=1.6)$ and clinical $(M=8.8 ; S D=2.7)$ samples indicated in previous research (Connor et al., 2001; Seeley-Wait, Abbott, \& Rapee, 2009). Of the initially recruited participants, 23 were excluded from analyses (see data analytic section for preregistered exclusion criteria).

The final sample of 131 participants self-reported their gender identity as $63.40 \%$ female, $32.10 \%$ male, and $4.60 \%$ non-binary. Participants ages ranged from $18-35(M=20.84, S D=$ 3.17) and they completed 14.38 years of education on average. Self-reported racial identities were as follows: $72.50 \%$ White, $11.50 \%$ Asian, 3.80\% Black or African American, 1.50\% 
Middle Eastern, $0.80 \%$ American Indian, $0.80 \%$ Native Hawaiian, and 9.20\% missing. A total of $11.50 \%$ of the sample reported Hispanic or Latinx ethnicity. A majority of the sample reported no previous psychological treatment $(53.40 \%)$.

\section{Measures}

Social Anxiety Symptoms. The Mini-SPIN (Connor et al., 2001) is a self-report screening measure for social anxiety. In accordance with previous research, we utilized the MiniSPIN to recruit participants with high and low levels of social anxiety (Seeley-Wait, Abbott, \& Rapee, 2009). Participants responded to this 3-item measure on a 0 (Not at all) to 4 (Extremely) Likert-type scale at two time points: Time 1 was conducted at recruitment and Time 2 was conducted at baseline (i.e., beginning of the study). Higher scores are indicative of greater social anxiety (Connor et al., 2001). Previous research has demonstrated excellent reliability and validity for the Mini-SPIN (Connor et al., 2001; Seeley-Wait, Abbott, \& Rapee, 2009). After data cleaning (see below), internal consistency for the Mini-SPIN in the current sample was strong at Time 1 and Time 2 (both $\alpha$ 's $=.88$ ). A paired-samples t-test was conducted with both timepoints and revealed no significant differences in Mini-SPIN scores from Time 1 to Time 2, $t(130)=1.27, p=.21$, demonstrating stability of Mini-SPIN scores.

State Anxiety. The Subjective Units of Distress Scale (SUDS; Coles \& Heimberg, 2000; Craske, 1999) assesses state anxiety on a 0 -100 scale. SUDS anchors were taken from Laborda et al. (2016), where a score of 0 indicates "not really experiencing anxiety, or barely noticeable anxiety", 25 indicates "mild anxiety, but it does not interfere with what you are doing", 50 indicates "uncomfortable anxiety level and concentration is somewhat affected", 75 indicates "uncomfortable anxiety that you are preoccupied with", and 100 indicates "the highest anxiety you have ever experienced or could imagine experiencing” (See Appendix). 
Lure Discrimination Index (LDI). The LDI is the measure of mnemonic discrimination abilities derived by the MST (Kirwan \& Stark, 2007). The LDI index controls for "similar" response biases (i.e., participants simply categorizing photographs as "similar" when uncertain). Lower LDI scores indicate poorer mnemonic discrimination abilities.2

Corrected Memory Recognition (REC). The REC is a traditional recognition memory index that reflects the ability to recognize old (i.e., targets) from new photographs (i.e., foils) on the MST (Kirwan \& Stark, 2007). The REC score is the "difference between the rate of 'old' responses given to the target items minus the corresponding rate of 'old' responses given to the foils” (cf: Stark, Kirwan, \& Stark, 2019). Lower scores indicate worse performance.

\section{Stressor Manipulation}

We experimentally manipulated the presence of a stressor (i.e., whether a participant is informed about the delivery of a future speech) prior to the encoding and retrieval phases of the MST. Specifically, at study onset, participants with high and low levels of social anxiety were randomized to either the stressor condition (i.e., learned of a future speech task prior to the MST) or the control condition (i.e., did not learn of a future speech task until the time of the speech task). Participants in the stressor condition were reminded about the speech task prior to the retrieval phase of the MST to reinstate the stressor (see Balderston et al., 2017).

\section{Tasks}

MST. The MST is a computerized paradigm used to assess mnemonic discrimination and recognition memory abilities. During the encoding phase (5 minutes), participants are shown a series of 128 photographs of everyday objects (i.e., candle, apple) and are instructed to categorize each photograph as an indoor or an outdoor item. During the retrieval phase (8 minutes) participants are shown a series of 192 photographs that include 64 objects that are 
repeated from the encoding phase (i.e., targets), 64 objects that are similar to the photographs in the encoding phase (i.e., lures), and 64 new objects (i.e., foils). Participants are provided with examples for each of the categories prior to categorizing the photographs presented as either new, similar, or old. All images in the encoding and retrieval phases were presented for 2 seconds with an inter-stimulus interval of 0.5 seconds.

Behavioral Avoidance Task (BAT). Following the MST, all participants were asked to give a speech about the topic "If I had a mission statement it would be...". Following Wong and Moulds (2009), participants were instructed: "You will now give a five-minute speech on your personal mission statement. This speech will be video recorded and may be reviewed by a psychologist, graduate students, and/or undergraduate students that are involved in this research laboratory. You will not be able to review your notes. Please stand in front of the video camera. You can stop this part of the experiment at any point. There is no right or wrong amount of time to participate in this task and it is not a test of courage. If you want to stop, please tell me to "stop." The video camera will continue recording unless you say stop. Do you have any questions?" Research assistants were instructed to record the speeches with a neutral expression and eye contact that naturally shifted from the video camera to the participant throughout the duration of the speech. Speech lengths were recorded on a de-identified record sheet.

\section{Procedure}

After consenting, participants responded to a series of demographic questions and measures for a larger study. Next, participants were informed about SUDS ratings and were provided with the anchors (see Appendix) to help describe their current experience of state anxiety. Research assistants then took their baseline SUDS ratings (SUDS 1). All participants were provided with an explanation of a personal mission statement (i.e., "a statement involves 
the aims and values of an individual for their life") and were allowed sixty seconds to take notes on their personal mission statements. At this time, participants in the stressor condition were informed of the speech task at the end of the study and were asked to take notes in preparation for that speech. Then, all participants were asked to provide another SUDS rating (SUDS 2). After, all participants completed the encoding phase of the MST and following the task, and they were asked to give provide a SUDS rating (SUDS 3). All participants were then instructed that they would be given an additional sixty seconds to take notes. Participants in the stressor condition were reminded of the impending speech task. All participants provided another SUDS rating (SUDS 4) prior to and after (SUDS 5) completing the retrieval phase of the MST.

Following the MST, participants were either informed (control condition) or reminded (stressor condition) of the speech task. After the video recorded speech, all participants were asked for their peak SUDS ratings during the speech task (SUDS 6). Lastly, all participants were debriefed, provided with treatment referrals (i.e., Carruth center), compensated either $\$ 10$ or 1 SONA credit, and thanked for their time.

\section{Data Analytic Plan}

All data exclusions were preregistered with OSF (https://osf.io/gc2d8). Participants were excluded from analyses if: 1) Self-reported ages were younger than 18 or older than $35(\mathrm{n}=2)$;

2) REC scores were below $50 \%(\mathrm{n}=8)$; 3) Mini-SPIN scores at Time 2 (baseline) put participants in a different group (i.e., high/low social anxiety) than Time 1 (recruitment; $\mathrm{n}=10$ );

4) Mini-SPIN scores at Time 1 and Time 2 had a difference greater than 3 points $(n=1) ; 5)$

Mini-SPIN scores were missing at Time 2 due to technological difficulties $(n=2)$. Final analyses included 131 participants (which allowed for analyses to find medium effect size; Faul, Erdfelder, Buchner, \& Lang, 2009) with roughly equal numbers of participants across study 
groups: high social anxiety stressor group $(n=33)$, high social anxiety control group $(n=33)$, low social anxiety stressor group $(n=33)$, low social anxiety control group $(n=32)$.

We conducted three primary analyses: 1) To assess whether recognition memory performance is correlated with lure discrimination indices, we ran a Pearson's correlation; 2) To determine the effectiveness (i.e., SUDS greater in high social anxiety) and stability (i.e., SUDS greater in stressor conditions from time 2 through 5) of stressor manipulations, we ran a 2 (group: high vs low social anxiety) x 2 (condition: stressor vs. control) x 6 (SUDS timepoints) mixed ANOVA; 3 ) To assess for differences in LDI scores, we ran a 2 (group: low vs. high social anxiety) x 2 (condition: stressor vs. control) between groups ANCOVA with REC as the covariate1.

\section{Results}

Preliminary t-test and chi-squared analyses were run to determine if there were significant differences across groups in demographic characteristics. There were no significant differences for gender $(p=.74)$, race $(p=.17)$, or ethnicity $(p=.40)$. However, age was significantly higher in the high social anxiety and no stressor condition $(p=.035)$ than the other groups. Analyses were re-run with age as a covariate, and the pattern of results remained unchanged, so the preregistered analyses are reported below.

Memory Recognition and Mnemonic Discrimination. As expected, a Pearson correlation indicated a significant positive association between REC and LDI scores, $r(131)=.868, p<$ .001 .

Stressor Stability and Effectiveness. A mixed ANOVA revealed a significant interaction between SUDS and condition (i.e., stressor or control), $F(5,635)=6.32, p<.001$, partial $\eta^{2}=$ .05 and between SUDS and group (i.e., high or low social anxiety), $F(5,635)=5.70, p<.001$, 
partial $\eta_{2}=.04$. To explore these interactions, we conducted separate independent samples ttests for condition and group effects on SUDS scores.

As expected, there were no significant differences of condition prior to stressor introduction (i.e., time $1 ; p=.42$ ) or after the speech task (i.e., time $6 ; p=.88$ ). During the stressor induction, SUDS scores significantly differed at time 2 (immediately after stressor induction), such that the stressor conditions had significantly higher SUDS than the control conditions, $t(129)=3.10, p<.001$. SUDS scores also significantly differed at time 4 (immediately after stressor re-induction), such that the stressor conditions had significantly higher SUDS than the control conditions, $t(129)=1.11, p=.01$. However, incongruent with our hypotheses, SUDS did not significantly differ at times 3 (immediately after the encoding phase of the MST; $p=.18$ ) or 5 (immediately after the retrieval phase of the MST; $p=.27$; see Figure 2).

Across anxiety groups, SUDS scores significantly differed at all time points. Individuals in the high social anxiety group had significantly higher SUDS scores than individuals in the low social anxiety group (all p's $<.001$; see Figure 3).

Lure Discrimination Scores. A between-subjects ANCOVA with REC scores as a covariate revealed nonsignificant effects of condition, $F(1,126)=.91, p=.34$, partial $\eta_{2}=.00$, and group, $F(1,126)=.60, p=.44$, partial $\eta_{2}=.00$. There was not a condition by group interaction, $F(1,126)=1.60, p=.21$, partial $\eta_{2}=.01($ see Figure 1$)$.

\section{Discussion}

The study reported herein aimed to elucidate the effects of state anxiety and social anxiety symptoms on mnemonic discrimination abilities. To do so, we utilized an analog sample of individuals with high and low levels of social anxiety and manipulated levels of state anxiety 
using a clinically relevant stressor task (i.e., knowledge of the delivery of a future speech) prior to assessing mnemonic discrimination abilities. We predicted that individuals in the stressor conditions would have worse mnemonic discrimination abilities than those in the control conditions. We also predicted that individuals with high levels of social anxiety in the stressor condition would have worse mnemonic discrimination abilities than those with high levels of social anxiety in the control condition. However, our results revealed no significant main or interactive effects of stressor condition or social anxiety group on mnemonic discrimination abilities.

Our findings are mixed in relation to previous research. Our data are consistent with past findings that anxiety symptoms on their own do not appear to be related to mnemonic discrimination abilities (Bernstein, Kleinman, \& McNally, 2019; Bernstein \& McNally, 2018). Although research is needed in diagnosed clinical samples, the reliable pattern of nonsignificance may suggest further explorations on symptoms alone to be a futile effort. Alternatively, our data are inconsistent with previous research suggesting the importance of state affect on mnemonic discrimination abilities (Balderston et al., 2017; Bernstein, Kleinman, \& McNally, 2019; Bernstein \& McNally, 2018). Further considerations of stressor assessments across studies are necessary to elucidate these discrepant findings.

In the current study, we hypothesized that state anxiety (as measured by SUDS) would be significantly different for the stressor and control conditions immediately after stressor induction (i.e., SUDS 2) through the end of the MST (i.e., SUDS 5). In doing so, our methods would align with previous research indicating a need for a threatening environment at both encoding and retrieval phases of the MST to negatively affect mnemonic discrimination abilities (Balderston et al., 2017). However, self-reported state anxiety scores were only higher in the stressor conditions 
immediately at post-stressor induction (SUDS 2) and reinstatement (SUDS 4) but not after the encoding and retrieval phases (i.e., SUDS 3 and SUDS 5). The instability of our stressor's effects (i.e., the variable levels of state anxiety for the stressor condition across hypothesized timepoints) may have resulted in less state anxiety during the actual MST phases. The variability of anxiety during the MST may not have been enough to negatively influence mnemonic discrimination abilities. Unlike Bernstein and colleagues (2019) who utilized two different stressors (via the trier social stress task; one prior to encoding and the other prior to retrieval), we utilized a single stressor (i.e., knowledge of a future speech) that was reintroduced in an effort to maintain its effect. However, their ability to maintain stressor effects across both phases of the MST may be likened to their use of multiple stressors in a single testing session. Likewise, it may be the case that state anxiety needs to be upheld during the phases of the MST (not just prior) to see effects on mnemonic discrimination. Future research should examine the dose and types of stressors needed to see such an impact.

Additionally, although we saw expected impacts of the stressor manipulation for socially anxious individuals compared to those with low levels of social anxiety, our stressor may not have provoked enough anxiety to allow for a significant interactive effect of state anxiety and social anxiety symptoms. Specifically, in individuals for whom we would expect to see the highest levels of state anxiety (i.e., those in the high social anxiety stressor condition), their average SUDS ratings were below 40 during all time points of the stressor manipulation (i.e., SUDS 2 through 5). Comparably, research on SUDS variability across exposure therapy for individuals with social anxiety disorder indicates an average starting point of $50-55$ (with higher baseline SUDS ratings for every one-point increase in pre-treatment symptom severity; Hayes, Hope, \& Heimberg, 2008). These data suggest that individuals with diagnosed social 
anxiety disorder may have had stronger responses to our clinically relevant stressor than our analog sample. Moreover, higher SUDS ratings map onto greater cognitive and physiological interference (i.e., SUDS anchor of 50: "uncomfortable anxiety level and your concentration is somewhat affected"). As such, SUDS scores below the scale midpoint may be too low to cause the inhibiting effects of state anxiety on mnemonic discrimination abilities. Thus, further examination of anxiety symptoms and state anxiety may require the use of clinical samples or more anxiety-inducing stressors to allow for interaction effects.

Another possibility for the lack of significant effects in the present study may be due to methodological differences in the way studies assessed reactions to the stressor. Both studies by Bernstein and colleagues $(2018,2019)$ utilized the Self-Assessment Manikin and combined scores of negative valence and emotional arousal to identify participants in a "state negative affect," (which they referred to as "state anxiety"). However, when arousal and negative affect are combined, the resulting experience includes a mixed state of depression (i.e., unhappy, melancholic, despaired) and anxiety (i.e., jittery, aroused, frenzied; Bradley \& Lang, 1994). Similarly, Balderston et al. (2017) found that mnemonic discrimination abilities were negatively impacted for participants who reported increased anxiety and fear and decreased positive affect. These findings may suggest the importance of a more broadly defined negative affective experience on mnemonic discrimination abilities (as opposed to state anxiety, specifically). Furthermore, it is possible that a combination of anxious and depressive symptoms influences mnemonic discrimination in a way that anxiety alone does not, and future research is needed to parse these effects.

Our current data do not provide evidence that state anxiety is related to mnemonic discrimination abilities. Additionally, across studies, trait anxiety does not appear to be related to 
mnemonic discrimination (Bernstein, Kleinman, \& McNally, 2019; Bernstein \& McNally, 2018).

Taken together, these data may indicate gaps in our knowledge about neuroscientific and theoretical links between fear overgeneralization for anxiety disorders and deficits in pattern separation processes. In short, anxiety disorders may not be as related to mnemonic discrimination as once expected. We are currently missing critical, empirical links between fear generalization and mnemonic discrimination in both healthy and anxious samples. Such behavioral data (supplemented with neuroimaging) would provide information about how and for whom the effects of impaired mnemonic discrimination should occur. It is possible that without this data, we are taking leaps in interpreting the interrelatedness of constructs, and in doing so, are missing important neurobiological or psychological evidence that differentiates these processes from one another.

Alternatively, it is possible that the role of mnemonic discrimination is relevant for anxiety disorders, but that this relation is mediated or moderated by other factors. At the moment, the data seem to suggest potential critical roles of trait depression (Dery et al., 2013; Shelton \& Kirwan, 2013) and state negative affect (i.e., state anxiety and depressive symptoms; Balderston et al., 2017; Bernstein, Kleinman, \& McNally, 2019; Bernstein \& McNally, 2018). However, there are likely other psychological and neurological factors that further explain how and why mnemonic discrimination and anxiety disorders are linked (or not). Nonetheless, the available data are too limited to draw any theoretical conclusions. At this stage, there is value in increasing collaboration efforts between clinical scientists and neuroscientists to define overlaps and missing links between overgeneralization, mnemonic discrimination, and pattern separation.

In addition to providing data on the effects of state anxiety, social anxiety, and mnemonic discrimination, the current study had multiple strengths. In particular, all data analytic 
procedures and hypotheses were preregistered, we utilized various time-points to determine the stability of the state anxiety manipulation, we had a robust number of participants compared to previous studies, and we used random assignment to determine stressor conditions. However, important limitations must also be considered. For one, we utilized an analog sample, which, as previously mentioned, may have influenced our ability to find effects. Assessing the clinically relevant stressor task in a sample of individuals with diagnosed social anxiety disorder may have strengthened the effects of the stressor manipulation and revealed a significant condition by group interaction on mnemonic discrimination abilities. Further, although the purpose of the study was blind to participants, our sample was self-selected (i.e., enrolled by responding to emails informing them of their eligibility). Due to this self-selection, participants in the current sample may differ in some meaningful way from a randomly selected sample (i.e., self-selection bias). Moreover, we did not assess for certain participant characteristics that may have impacted the validity of our findings (i.e., head injury, cognitive impairment, neurologic disorder, mania, or psychosis). Participant selection and subsequent randomization was also only single-blind (i.e., research assistants knew whether their participants had high or low levels of social anxiety), so research assistants may have unknowingly provided differential treatment of individuals according to their symptomatic status in a way that influenced results. We also did not include any physiological assessments of anxiety or any assessments of depressive symptoms, which may have provided more nuanced information about the impact of our stressor across study groups.

\section{Conclusion}

Based on the data from the present sample, it does not appear that state anxiety or social anxiety symptoms impact mnemonic discrimination abilities. However, this may be explained by 
the instability of our stressor or generally low levels of state anxiety experienced during the stressor manipulation. Previous research may also have captured a distinct affective experience from state anxiety, in which depressive symptoms interact with symptoms of anxiety to negatively affect mnemonic discrimination. Future research should focus on identifying clarifying the affective experiences that influence mnemonic discrimination abilities and the type, intensity, and duration of effective stressor-inductions. In addition to these mechanistic studies, empirical studies linking psychological theories and neuroscience (i.e., overgeneralization, mnemonic discrimination, and pattern separation) must be carried out in healthy and clinically anxious samples to provide a better framework for current findings. These vital studies and collaborations between clinical scientists and neuroscientists will ultimately determine the future directions of mnemonic discrimination research for anxiety disorders. 


\section{Footnotes}

1 In accordance with previous research (Bernstein, Kleinman, \& McNally, 2019; Bernstein \& McNally, 2018), REC was included as a covariate to isolate mnemonic discrimination abilities beyond general memory capacity.

2 LDI scores can range from -1 to +1 (or -100 to 100 , depending on author reporting preference). Although, negative LDI scores are usually removed with data cleaning procedures that are standard for the MST (to ensure data quality). In the current study, all negative scores $(\mathrm{n}=3)$ were removed with the data cleaning procedures listed in the manuscript. 


\section{References}

Ahrens, L. M., Mühlberger, A., Pauli, P., \& Wieser, M. J. (2014). Impaired visuocortical discrimination learning of socially conditioned stimuli in social anxiety. Social Cognitive and Affective Neuroscience, 10, 929-937. doi:10.1093/scan/nsu140

American Psychiatric Association (2013). Diagnostic and Statistical Manual of Mental Disorders (5th ed.). Arlington, VA: American Psychiatric Publishing.

Bakker, A., Krauss, G. L., Albert, M. S., Speck, C. L., Jones, L. R., Stark, C. E., ... \& Gallagher, M. (2012). Reduction of hippocampal hyperactivity improves cognition in amnestic mild cognitive impairment. Neuron, 74, 467-474. doi:10.1016/j.neuron.2012.03.023

Balderston, N. L., Mathur, A., Adu-Brimpong, J., Hale, E. A., Ernst, M., \& Grillon, C. (2017). Effect of anxiety on behavioural pattern separation in humans. Cognition and Emotion, 31, 238-248. doi:10.1080/02699931.2015.1096235

Bannerman, D. M., Rawlins, J. N. P., McHugh, S. B., Deacon, R. M. J., Yee, B. K., Bast, T., ... \& Feldon, J. (2004). Regional dissociations within the hippocampus-memory and anxiety. Neuroscience \& Biobehavioral Reviews, 28(3), 273-283. doi:10.1016/j.neubiorev.2004.03.004

Bernstein, E. E., Kleiman, E. M., \& McNally, R. J. (2019). Mnemonic Discrimination Under Stress and Its Clinical Relevance for Anxiety. Clinical Psychological Science, 1 - 18. doi:10.1177/216770261983456

Bernstein, E. E., \& McNally, R. J. (2018). Exploring behavioral pattern separation and risk for emotional disorders. Journal of Anxiety Disorders, 59, 27-33. doi:10.1016/j.janxdis.2018.08.006 
Bradley, M. M., \& Lang, P. J. (1994). Measuring emotion: the self-assessment manikin and the semantic differential. Journal of Behavior Therapy and Experimental Psychiatry, 25, 49-59. doi:10.1016/0005-7916(94)90063-9

Clemenson, G. D., Henningfield, C. M., \& Stark, C. (2019). Improving hippocampal memory through the experience of a rich Minecraft environment. Frontiers in Behavioral Neuroscience, 13, 57. doi:10.3389/fnbeh.2019.00057

Clemenson, G. D., \& Stark, C. E. (2015). Virtual environmental enrichment through video games improves hippocampal-associated memory. Journal of Neuroscience, 35, 1611616125. doi:10.1523/JNEUROSCI.2580-15.2015

Coles, M. E., \& Heimberg, R. G. (2000). Patterns of anxious arousal during exposure to feared situations in individuals with social phobia. Behaviour Research and Therapy, 38(4), 405424. doi:10.1016/S0005-7967(99)00092-3

Connor, K. M., Kobak, K. A., Churchill, L. E., Katzelnick, D., \& Davidson, J. R. (2001). MiniSPIN: A brief screening assessment for generalized social anxiety disorder. Depression and Anxiety, 14, 137-140. doi:10.1002/da.1055

Craske, M. G. (1999). Anxiety disorders: Psychological approaches to theory and treatment. Boulder, CO: Westview Press.

Craske, M. G., Rauch, S. L., Ursano, R., Prenoveau, J., Pine, D. S., \& Zinbargh, R. E. (2009). What is an anxiety disorder? Depression and Anxiety, 26, 1066-1085. doi:10.1002/da.20633

Das, T., Ivleva, E. I., Wagner, A. D., Stark, C. E., \& Tamminga, C. A. (2014). Loss of pattern separation performance in schizophrenia suggests dentate gyrus dysfunction. Schizophrenia Research, 159, 193-197. doi:10.1016/j.schres.2014.05.006 
DeCarolis, N. A., \& Eisch, A. J. (2010). Hippocampal neurogenesis as a target for the treatment of mental illness: a critical evaluation. Neuropharmacology, 58(6), 884-893. doi:10.1016/j.neuropharm.2009.12.013

Dunsmoor, J. E., \& Paz, R. (2015). Fear generalization and anxiety: behavioral and neural mechanisms. Biological Psychiatry, 78(5), 336-343.

Dymond, S., Dunsmoor, J. E., Vervliet, B., Roche, B., \& Hermans, D. (2015). Fear generalization in humans: systematic review and implications for anxiety disorder research. Behavior Therapy, 46, 561-582. doi:10.1016/j.beth.2014.10.001

Endler, N. S., \& Kocovski, N. L. (2001). State and trait anxiety revisited. Journal of Anxiety Disorders, 15(3), 231-245. doi:10.1016/S0887-6185(01)00060-3

Faul, F., Erdfelder, E., Buchner, A., \& Lang, A.-G. (2009). Statistical power analyses using G*Power 3.1: Tests for correlation and regression analyses. Behavior Research Methods, 41, 1149-1160.

Fujii, T., Saito, D. N., Yanaka, H. T., Kosaka, H., \& Okazawa, H. (2014). Depressive mood modulates the anterior lateral CA1 and DG/CA3 during a pattern separation task in cognitively intact individuals: a functional MRI study. Hippocampus, 24, 214-224. doi:10.1002/hipo.22216

Gray, J. A. (1987). The psychology of fear and stress (Vol. 5). CUP Archive.

Hayes, S. A., Hope, D. A., \& Heimberg, R. G. (2008). The pattern of subjective anxiety during in-session exposures over the course of cognitive-behavioral therapy for clients with social anxiety disorder. Behavior Therapy, 39(3), 286-299.

doi:10.1016/j.beth.2007.09.001 
Hermann, C., Ziegler, S., Birbaumer, N., \& Flor, H. (2002). Psychophysiological and subjective indicators of aversive Pavlovian conditioning in generalized social phobia. Biological Psychiatry, 52, 328-337. doi:10.1016/S0006-3223(02)01385-9

Irle, E., Ruhleder, M., Lange, C., Seidler-Brandler, U., Salzer, S., Dechent, P., ... \& Leichsenring, F. (2010). Reduced amygdalar and hippocampal size in adults with generalized social phobia. Journal of Psychiatry \& Neuroscience: JPN, 35, 126 - 131. doi:10.1503/jpn.090041

Kheirbek, M. A., \& Hen, R. (2014). Add neurons, subtract anxiety. Scientific American, 311, 62-67. doi:10.1038/scientificamerican0714-62

Kirschbaum, C., Pirke, K. M., \& Hellhammer, D. H. (1993). The 'Trier Social Stress Test'-a tool for investigating psychobiological stress responses in a laboratory setting. Neuropsychobiology, 28(1-2), 76-81. doi:10.1159/000119004

Kirwan, C. B., Hartshorn, A., Stark, S. M., Goodrich-Hunsaker, N. J., Hopkins, R. O., \& Stark, C. E. (2012). Pattern separation deficits following damage to the hippocampus. Neuropsychologia, 50, 2408-2414. doi:10.1016/j.neuropsychologia.2012.06.011

Kirwan, C. B., \& Stark, C. E. (2007). Overcoming interference: an fMRI investigation of pattern separation in the medial temporal lobe. Learning \& Memory, 14, 625-633. doi:10.1101/lm.663507

Kraguljac, N. V., Carle, M., Frölich, M. A., Tran, S., Yassa, M. A., White, D. M., ... \& Lahti, A. C. (2018). Mnemonic discrimination deficits in first-episode psychosis and a ketamine model suggests dentate gyrus pathology linked to N-methyl-D-aspartate receptor 
hypofunction. Biological Psychiatry: Cognitive Neuroscience and Neuroimaging, 3(3), 231-238. doi:10.1016/j.bpsc.2017.02.005

Laborda, M. A., Schofield, C. A., Johnson, E. M., Schubert, J. R., George-Denn, D., Coles, M. E., \& Miller, R. R. (2016). The extinction and return of fear of public speaking. Behavior Modification, 40, 901-921. doi:10.1177/0145445516645766

Lacy, J. W., Yassa, M. A., Stark, S. M., Muftuler, L. T., \& Stark, C. E. (2011). Distinct pattern separation related transfer functions in human CA3/dentate and CA1 revealed using highresolution fMRI and variable mnemonic similarity. Learning \& Memory, 18, 15-18. doi:10.1101/lm.1971111

Lang, P. J., Greenwald, M. K., Bradley, M. M., \& Hamm, A. O. (1993). Looking at pictures: Affective, facial, visceral, and behavioral reactions. Psychophysiology, 30(3), 261-273.

Lange, I., Goossens, L., Michielse, S., Bakker, J., Lissek, S., Papalini, S., ... \& Lieverse, R. (2017). Behavioral pattern separation and its link to the neural mechanisms of fear generalization. Social Cognitive and Affective Neuroscience, 12, 1720-1729. doi:10.1093/scan/nsx104

Leal, S. L., \& Yassa, M. A. (2018). Integrating new findings and examining clinical applications of pattern separation. Nature Neuroscience, 21, 163-173.

Liao, W., Xu, Q., Mantini, D., Ding, J., Machado-de-Sousa, J. P., Hallak, J. E., ... \& Crippa, J. A. S. (2011). Altered gray matter morphometry and resting-state functional and structural connectivity in social anxiety disorder. Brain Research, 1388, 167-177. doi:10.1016/j.brainres.2011.03.018 
Lissek S. (2012). Toward an account of clinical anxiety predicated on basic, neurally mapped mechanisms of Pavlovian fear-learning: the case for conditioned overgeneralization. Depression and Anxiety, 29, 257-63. doi:10.1002/da.21922

Mannuzza, S., Schneier, F. R., Chapman, T. F., Liebowitz, M. R., Klein, D. F., \& Fyer, A. J. (1995). Generalized social phobia: Reliability and validity. Archives of General Psychiatry, 52, 230-237. doi:10.1001/archpsyc.1995.03950150062011

Ponzini, G. T., \& Steinman, S. (2019, October 4). Mnemonic Discrimination and Social Anxiety: The Role of State Anxiety. Retrieved from osf.io/gc2d8

Qiu, C., Liao, W., Ding, J., Feng, Y., Zhu, C., Nie, X., ... \& Gong, Q. (2011). Regional homogeneity changes in social anxiety disorder: a resting-state fMRI study. Psychiatry Research: Neuroimaging, 194, 47-53. doi:10.1016/j.pscychresns.2011.01.010

Robinson, O. J., Letkiewicz, A. M., Overstreet, C., Ernst, M., \& Grillon, C. (2011). The effect of induced anxiety on cognition: threat of shock enhances aversive processing in healthy individuals. Cognitive, Affective, \& Behavioral Neuroscience, 11(2), 217. doi:10.3758/s13415-011-0030-5

Ruscio, A. M., Brown, T. A., Chiu, W. T., Sareen, J., Stein, M. B., \& Kessler, R. C. (2008). Social fears and social phobia in the USA: results from the National Comorbidity Survey Replication. Psychological Medicine, 38, 15-28. doi:10.1017/S0033291707001699

Sachs, G., Anderer, P., Doby, D., Saletu, B., \& Dantendorfer, K. (2003). Impaired conditional discrimination learning in social phobia. Neuropsychobiology, 47, 66-72. doi:10.1159/000070011 
Sahay, A., Scobie, K. N., Hill, A. S., O'Carroll, C. M., Kheirbek, M. A., Burghardt, N. S., ... \& Hen, R. (2011). Increasing adult hippocampal neurogenesis is sufficient to improve pattern separation. Nature, 472, 466. doi:10.1038/nature09817

Seeley-Wait, E., Abbott, M. J., \& Rapee, R. M. (2009). Psychometric properties of the minisocial phobia inventory. Primary Care Companion to the Journal of Clinical Psychiatry, 11, 231. doi:10.4088/PCC.07m00576

Segal, S. K., Stark, S. M., Kattan, D., Stark, C. E., \& Yassa, M. A. (2012). Norepinephrinemediated emotional arousal facilitates subsequent pattern separation. Neurobiology of Learning and Memory, 97(4), 465-469. doi:10.1016/j.nlm.2012.03.010

Shelton, D. J., \& Kirwan, C. B. (2013). A possible negative influence of depression on the ability to overcome memory interference. Behavioural Brain Research, 256, 20-26. doi:10.1016/j.bbr.2013.08.016

Stark, S. M., Kirwan, C. B., \& Stark, C. E. (2019). Mnemonic Similarity Task: A Tool for Assessing Hippocampal Integrity. Trends in Cognitive Sciences. doi:10.1016/j.tics.2019.08.003

Stark, S. M., Stevenson, R., Wu, C., Rutledge, S., \& Stark, C. E. (2015). Stability of age-related deficits in the mnemonic similarity task across task variations. Behavioral Neuroscience, 129, 257-268. doi:10.1037/bne0000055

Stark, S. M., Yassa, M. A., Lacy, J. W., \& Stark, C. E. (2013). A task to assess behavioral pattern separation (BPS) in humans: Data from healthy aging and mild cognitive impairment. Neuropsychologia, 51, 2442-2449.

doi:10.1016/j.neuropsychologia.2012.12.014 
Stein, D. J., Lim, C. C., Roest, A. M., de Jonge, P., Aguilar-Gaxiola, S., Al-Hamzawi, A., ... \& de Girolamo, G. (2017). The cross-national epidemiology of social anxiety disorder: Data from the World Mental Health Survey Initiative. BMC Medicine, 15, 143-164. doi: $10.1186 / \mathrm{s} 12916-017-0889-2$

Wong, Q. J. (2016). Anticipatory Processing and Post-Event Processing in Social Anxiety Disorder: An Update on the Literature. Australian Psychologist, 51, 105-113. doi:10.1111/ap.12189

Wong, Q. J., \& Moulds, M. L. (2009). Impact of rumination versus distraction on anxiety and maladaptive self-beliefs in socially anxious individuals. Behaviour Research and Therapy, 47(10), 861-867. doi:10.1016/j.brat.2009.06.014

Yassa, M. A., \& Stark, C. E. (2011). Pattern separation in the hippocampus. Trends in Neurosciences, 34, 515-525. doi:10.1016/j.tins.2011.06.006

Yassa, M. A., Mattfeld, A. T., Stark, S. M., \& Stark, C. E. (2011). Age-related memory deficits linked to circuit-specific disruptions in the hippocampus. Proceedings of the National Academy of Sciences, 108, 8873-8878. doi:10.1073/pnas.1101567108

Yassa, M. A., Stark, S. M., Bakker, A., Albert, M. S., Gallagher, M., \& Stark, C. E. (2010). High-resolution structural and functional MRI of hippocampal CA3 and dentate gyrus in patients with amnestic mild cognitive impairment. Neuroimage, 51, 1242-1252. doi:10.1016/j.neuroimage.2010.03.040 


\section{Figures}

Figure 1

Means and Standard Deviations for LDI Scores Across Study Groups

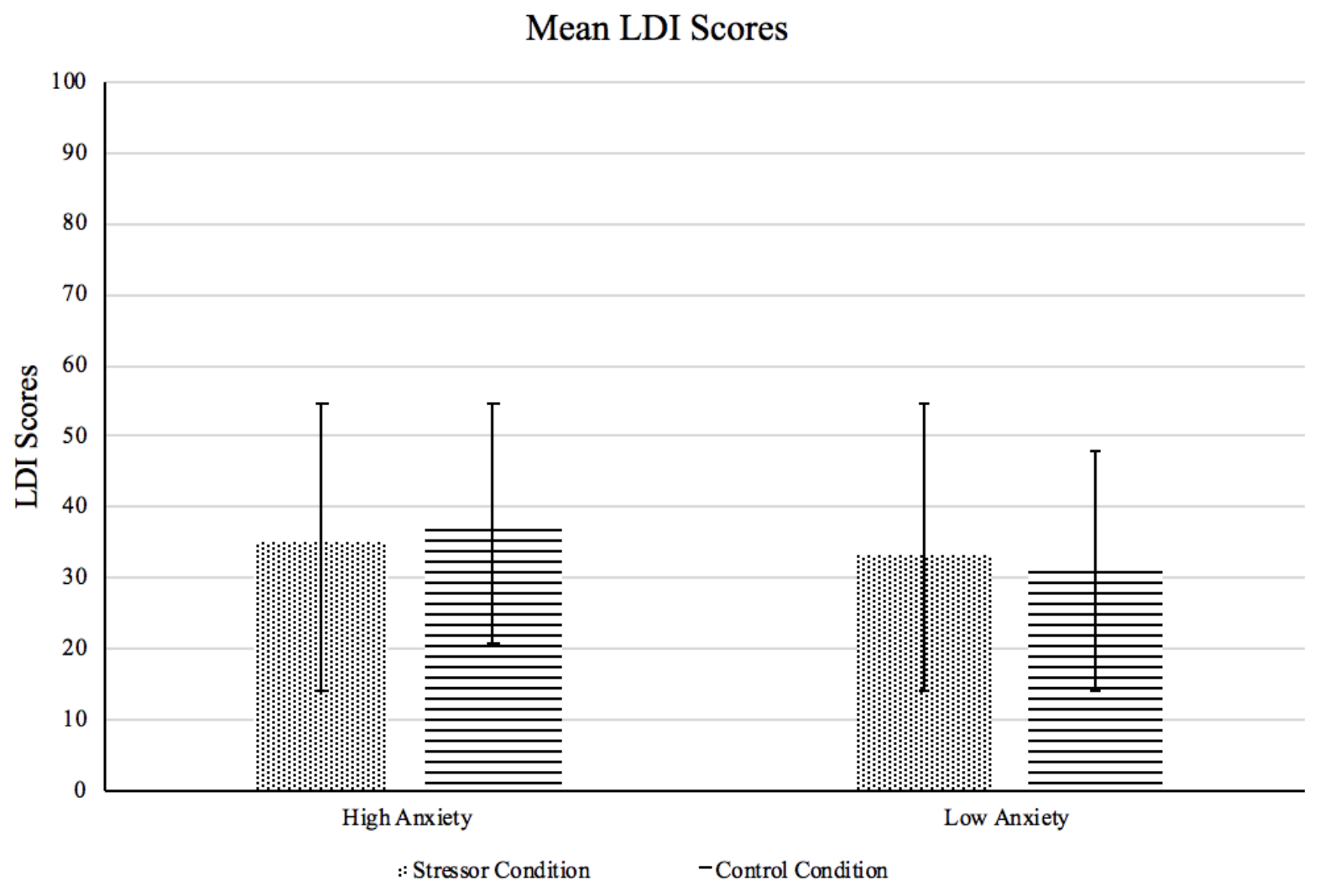


Figure 2

Means and Standard Errors for SUDS Scores by Condition

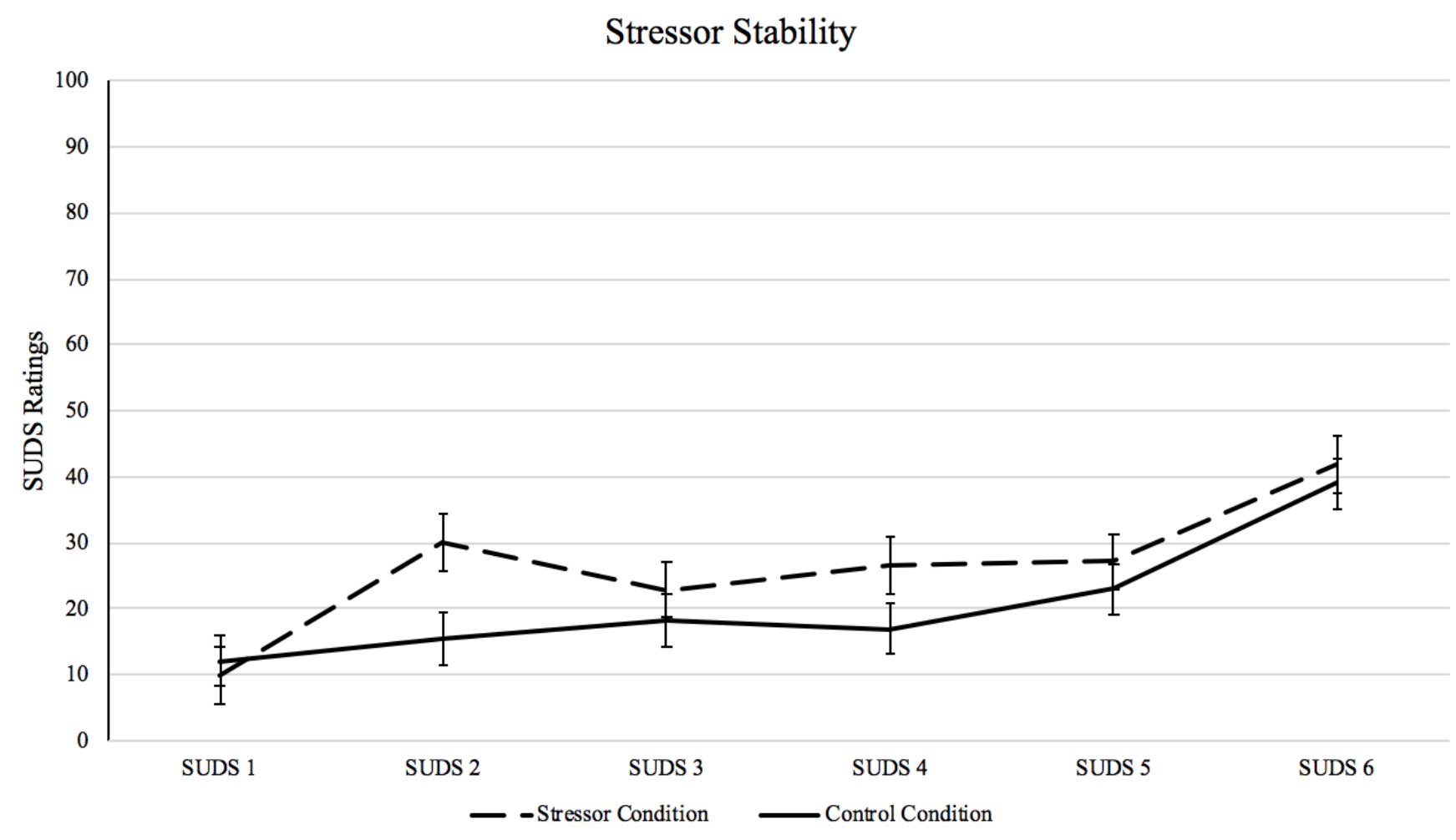


Figure 3

Means and Standard Errors for SUDS Scores by Group

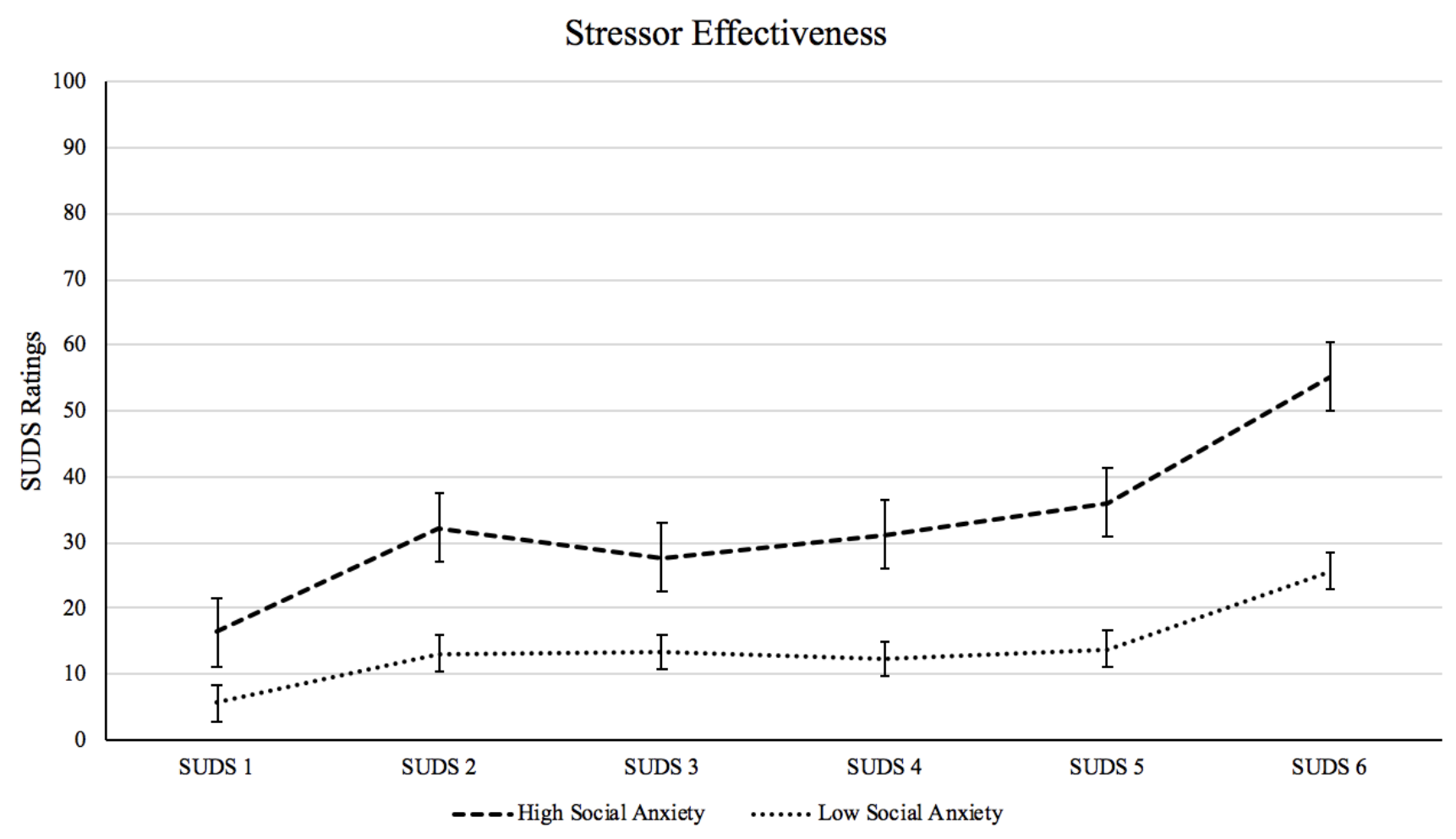

